\title{
Principles of Encoding and Methods of Measurement for the Provision of Navigation Systems of Maritime Application
}

\author{
Valentin Chimshir ${ }^{1}$, Andrey Lysyy ${ }^{2}$, Oleksandr Danylenko $^{3}$, Eugene Panarkin ${ }^{2}$, Olena Soroka ${ }^{3}$, Inna Skliarenko $^{4}$ \\ ${ }^{1}$ Director of the Institute, Danube Institute of National University of “Odessa Maritime Academy”, Ukraine \\ scopus667@gmail.com \\ ${ }^{2}$ Department of «Navigation and Ship Management» Azov Maritime Institute National University "Odessa Maritime Academy", Ukraine, \\ anlis@ami.edu.ua, evp110656@gmail.com \\ ${ }^{3}$ Department the Navigation and Ship Handling, Danube Institute of National University of “Odessa Maritime Academy”, Ukraine \\ scopus667@gmail.com, elkazej@gmail.com \\ ${ }^{4}$ Department of technologies and operational processes in navigation, Hetman Petro Konashevych-Sahaidachnyi Kyiv Institute of Water \\ Transport of the State University of Infrastructure and Technologies, Ukraine,
}

\begin{abstract}
The article fulfills and discusses the principles of GPS operation; analyzed the main operational characteristics of the satellite navigation system; investigated alternative ways of organizing a satellite radio navigation system in order to improve operational characteristics; the costs of operating a GPS satellite are calculated. Methods of generating codes for providing satellite navigation systems GPS / GLONASS are considered.
\end{abstract}

Key words: Navigation Systems, Errors, GPS, GLONASS, Positioning.

\section{INTRODUCTION}

To solve many problems, various types of receivers are used, but, no matter how different the scope of GPS applications, they all have one thing in common - the need for accurate determination of coordinates. It can be noted that the errors that lead to inaccuracies in determining the position when solving different problems are mostly the same. But the methods used to fix them are often very different. This is mainly due to the fact that we have different requirements for accuracy and conditions under which positioning occurs, and the quality and level of the receiver also play an important role. The capabilities of navigation systems will expand due to modernization, but for modernization it is necessary to outline the goals of improvement, that is, to analyze the operation of these systems and the factors affecting their operation. This shows the relevance of my work, as it is devoted to the analysis of the operational characteristics of satellite navigation systems and the influence on their operation of various factors.

The purpose of this article is to evaluate the application of the principles of the formation of code signals and measurement methods to provide satellite navigation systems.

Achieving this goal required setting and solving the following set of tasks:

consideration of the principles of GPS operation; analysis of errors leading to inaccuracies in the determination of coordinates, their magnitude, various methods of correction and the scope of these methods;

research and analysis of alternative ways of organizing radio navigation systems to reduce operating costs;

analysis and calculation of operating costs for navigation satellite systems.

To implement these tasks, the following methods were used in the work: analytical, expert, analysis method, graphic. All this predetermined the structure of this work, which consists of seven main sections.

The theoretical and methodological basis of the work was the works of domestic and foreign researchers on the theory of GPS, statistical data on the development of GPS measurements in modern conditions, scientific works, periodicals, articles.

\section{MAIN MATERIAL}

\section{P-code generation}

Formation of code signals is carried out in the generators of $\mathrm{P}$ and C / A-codes. Structurally, both generators are made on bit registers, the forming polynomials for which are set by the onboard processor. The GPS P-code generator consists of two twenty-four-bit registers, the bit state in which changes with a frequency of $10.23 \mathrm{MHz}$. Functionally, the generator works according to the time dependence:

$$
P(t)=P 1(t)+P 2(t+i T)
$$

Each of these registers, in turn, is structurally executed on the combined two twelve-bit registers. The registers are labeled accordingly.

$$
P 1 B=1+x^{1}+x^{2}+x^{5}+x^{8}+x^{9}+x^{10}+x^{11}+x^{12} .
$$

And the initial states of the registers have values:

$$
P 1 B \rightarrow 000100100100,
$$


Valentin Chimshir et al., International Journal of Emerging Trends in Engineering Research, 8(9), September 2020,5191 - 5196

$$
P 1 B \rightarrow 001010101010
$$

Generating polynomials for register P2 have the form:

$$
P 2 B=1+x^{1}+x^{2}+x^{3}+x^{4}+x^{8}+x^{9}+x^{12} .
$$

The initial states of the registers are determined by the sequences:

$$
\begin{aligned}
& P 2 A \rightarrow 101001001001, \\
& P 2 B \rightarrow 001010101010,
\end{aligned}
$$

The sequences are formed with a delay of an integer number of clock cycles $i=1 \ldots 37$, each of which is set for a specific satellite. Each sequence is summed modulo $2 \mathrm{~s}$ by the sequence. As a result, 37 unique sequences are formed, each of which belongs to a specific satellite. The number of states (bits) of the register is determined by the value 15345000 , which corresponds to a time interval of $1.5 \mathrm{~s}$. Every $1.5 \mathrm{~s}$, the states of the registers are repeated, but with new signs. When a new week starts, the shift registers P1A, P1B, P2A, P2B are initialized to generate the first digit of the week [1-3, 5, 7]. At the end of the week, a sign of the end of the week is formed.

\section{Formation of C / A-code}

The C / A code generator generates a signal intended for civilian consumers. To form the $\mathrm{C} / \mathrm{A}$ signal, a Gold polynomial with a period of $1 \mathrm{~ms}$, a clock frequency of 1.023 $\mathrm{MHz}$ was applied. The Gold polynomial is generated by the generator circuit.

The scheme uses two ten-bit shift registers with feedback, the forming sequences of which are set by the onboard central computer and are determined by polynomials, one of which is represented by the expression:

$$
G_{21}(x)=1+x^{2}+x^{3}+x^{6}+x^{8}+x^{9}+x^{10},
$$

The addition of these sequences forms the Gold code signal:

$$
X G_{i}(t)=G_{1}+G_{2}\left[t+M_{i}(10 T)\right]
$$

\section{Formation of the rangefinder code and GLONASS sync pulses}

The ranging code is a pseudo-random sequence with a length of 511 elements with a repetition period of 10-3 s. Shaping polynomial:

$$
G(x)=1+x^{5}+x^{9}
$$

\section{Formation of phase modulation of the carrier signal}

The carrier signal is modulated by two binary sequences, each of which is formed by summing modulo 2 the ranging code and the transmitted system and navigation data. The first sequence is the modulo 2 sum of the ranging $\mathrm{P}$-code and navigation data. The second sequence is the modulo 2 sum of the open C / A code and the same navigation data sequence.

To exclude the possible overlap of P- and C / A-code signals, the transmission is carried out by quadrature components:

sinusoidal;

cosine.

For this implementation, phase inverters "/ 2" are provided in the spacecraft circuit, which shift the phases of the carrier oscillations containing the P-code signal by $90^{\circ}$.

The radio signal at a frequency is biphasic keyed by only one of the two previously considered sequences.

The choice of the modulating sequence is provided by command from the ground control and command complex.

\section{Measurement methods when using satellite navigation systems}

\section{Absolute method}

The method is based on quasi-distance measurements. Their use is possible if the time scales of the clocks of all satellites in the system are synchronized, and the observer's clocks have sufficient stability during the measurement time. But the observer's time has a "departure" from the scale of the satellite system.

In this case, the multichannel receiver records the time intervals of the arrival of signals from several satellites relative to the local time scale. These intervals define the corresponding pseudo-ranges, which differ from the true ranges by an amount obtained by shifting the consumer's time scale relative to the satellite clock's time scale.

The delay time is defined as the difference between the clock readings of the satellite and the receiver at the moments of generation of the same signal:

$$
\tau(t)=t_{A}
$$

The satellite's motion is taken into account by the dependence of the measured values on time, sometimes the designation $t$ is omitted to shorten the record.

Measurements are performed by code or phase methods. Code methods are significantly inferior in accuracy to phase methods and are performed using the $\mathrm{C} / \mathrm{A}$ or P-code, and the most accurate measurements are performed by phase methods at carrier frequencies and

The main characteristics of codes and signals, for example, for GPS are presented in Table 1. 
Valentin Chimshir et al., International Journal of Emerging Trends in Engineering Research, 8(9), September 2020, 5191 - 5196

Table 1: Characteristics of GPS signals

\begin{tabular}{|c|c|c|c|c|}
\hline Параметры & Duration and length of code & Frequency, MHz & $\begin{array}{c}\text { Code symbol period } \\
\text { and wavelength }\end{array}$ & $\begin{array}{c}\text { Accuracy, } 1 \% \text { of } \\
\text { wavelength }\end{array}$ \\
\hline C/A- code & $1 \mathrm{~ms}$ & 1,023 & $1,0 \mu \mathrm{s}$ & $\begin{array}{c}10 \mathrm{~ns} \\
3,0 \mathrm{~m}\end{array}$ \\
\hline P- code & $293,26 \mathrm{~km}$ & $1,023,26 \mathrm{~m}$ & $0,1 \mu \mathrm{s}$ \\
& 7 cyтoK & & $29,33 \mathrm{~m}$ & $0,3 \mathrm{~m}$ \\
\hline & $18,1 * 10^{10} \mathrm{~km}$ & 1227,600 & $24,4 \mathrm{sm}$ & $2,4 \mathrm{~mm}$ \\
\hline & $19,2 \mathrm{sm}$ & 1575,420 & $19,05 \mathrm{sm}$ & $1,9 \mathrm{~mm}$ \\
\hline
\end{tabular}

From the data in Table 1, it follows that one character of the $\mathrm{C}$ / A code corresponds to approximately $293 \mathrm{~m}$, and the entire code corresponds to $293 \mathrm{~km}$. In other words, the C / A code is 1,000 characters long. The entire distance of more than 20,200 $\mathrm{km}$ from the satellite to the GPS-receiver will consist of $\mathrm{N}$ segments of $293.26 \mathrm{~km}$ each, $\mathrm{m}$ segments of $293.26 \mathrm{~m}$ each and a fraction of $296.26 \mathrm{~m}$.

The value $\mathrm{N}$ is an integer number of milliseconds of signal propagation from the satellite to the receiver, equal to about 70-80 units. It is impossible to fix the value of $\mathrm{N}$, it determines the ambiguity of C / A code measurements. It is found in a different way, for example, by the distance, approximately known with an accuracy of $100 \mathrm{~km}$. To do this, set the approximate coordinates of the receiver, determined from the map. The values of $m$ and are measured by aligning the signal generated in the receiver with the code received from the satellite by successive shift until the correlation occurs.

It is known that on the satellite and in the receiver, codes are generated strictly synchronously. Then the satellite signal arriving at the receiver will "lag" in proportion to the propagation time in relation to the receiver signal, and when superimposed, the codes will not match. The local signal of the receiver is sequentially "delayed" for such a measured time so that the received signal has time to "catch up", and they both coincide.

The quality of the result will depend on the accuracy of the code interpolation. Modern devices allow you to fix with an accuracy of 0.01 fraction of a period and even more accurately. In a linear measure, this amounts to about $3 \mathrm{~m}$ for the $\mathrm{C} / \mathrm{A}$ code. Therefore, if there were no other sources of errors, then the ranges could be measured with an error of several meters and more accurately. The exact P-code has higher capabilities. In GPS, the duration of the P-code symbol corresponds to a distance of $30 \mathrm{~m}$. The measurement accuracy will be 10 times higher than for the standard C / A code. The duration of the $\mathrm{P}$-code is 7 days, which is many times longer than the travel time of the radio wave path from the satellite to the GPS receiver. The time at the distance "satellite - receiver" is estimated at about $0.07 \mathrm{~s}$.

The number of characters in the P-code is about $6 * 1012 \mathrm{~s}$. The range is uniquely determined with an accuracy of about $0.3 \mathrm{~m}$. However, it is desirable to know the approximate value of the coordinates of the ground station. The latency time of the code is also unknown. A very long search time would be required for a full sequential enumeration of the duplicate P-code signals of the receiver until the moment of coincidence with a similar code sequence of the received signals. The receiver is able to "scan" in $1 \mathrm{~s}$ up to 1000 characters. When the coordinates of the station are not known at all, they are taken equal to zero. In this case, the receiver is located at the center of mass of the Earth. The distance from the satellite to the center of the Earth is always greater than to its surface, the largest error in the approximate distance will be equal to the radius of the Earth $(6,371 \mathrm{~km})$. It is easy to calculate that the search time will not exceed four minutes. Therefore, modern GPS-receivers find the location without prior indication of the current time and approximate coordinates of the station [8-13].

Four simultaneously measured pseudo-ranges, as well as the data on ephemeris and time parameters of the satellites' clocks, received in the navigation message, make it possible to calculate three coordinates of the observer and a correction to the time scale of the receiver relative to the scale of the spacecraft.

From experimental measurements it was found that at $=10^{18}$ $\mathrm{m}-2$ the delays are $9^{-12}$ meters at zenith angles near $90^{\circ}$, and at zenith angles of $15^{\circ}$, the delays increase threefold. The measurement errors in determining the ionospheric delay are 2 $\mathrm{m}$.

Currently, a more highly accurate method for determining the ionospheric delay is based on the use of two-frequency measurements. Due to the dispersion properties of the ionosphere for radio waves, the values of the "satellite receiver" path distances obtained from measurements at the carrier waves are different. From the measured ranges and parameters of the carrier frequencies, a "pure range" is obtained, free of ionospheric delay errors.

Air temperature, pressure and humidity are determined at the point where the receiver is located. The errors in determining tropospheric delays at zenith angles from 30 to $80^{\circ}$ are $1-2 \mathrm{sm}$ when applying the Saastamoinen formula.

\section{Differential method}

The differential method is designed to improve the accuracy of measurements of both pseudo-ranges and other parameters. The mode is based on the formation of differences in readings, therefore it is called differential. 
The signals from the satellites are sent to the consumer object, for example, an aircraft and to the control station. At the control station, in the precision equipment of the receiver, the measurement results are accumulated, and random errors are filtered in them. This data is sent to the correcting information generator. For correction, the information is compared with the precise data of the control station, obtained by means of geodetic referencing. The generated corrective corrections are sent through the transmitter to the consumer's object.

This mode reveals the systematic parts of the errors of the ephemeris, the drift of the time scale, the influence of the ionosphere and troposphere. The effect of using the method depends on the degree of spatial and temporal correlations between the control station and the consumer object. With a strong correlation, the systematic part of the errors will be completely eliminated.

The differential method uses the following methods for entering corrective corrections:

coordinate correction;

correction of navigation corrections.

\section{Coordinate correction}

The method assumes that the correcting information is generated at the control station by comparing the reference coordinates (the antenna coordinates are determined from geodetic data) with the coordinates calculated as a result of a navigation session conducted by the consumer's equipment of the highest accuracy class. The resulting differential corrections are transmitted to the consumer object, which specifies its coordinates based on them.

The disadvantage of this method is the limitation of the range. The consumer works according to the most advantageous form of the constellation of satellites (GDOP factor), and the control station should work according to the same constellation. This is possible at small distances from the control station (hundreds of kilometers). The transfer of corrections found for one constellation to the results of determinations for another constellation degrades the accuracy of measurements.

\section{Correction of navigation parameters}

The peculiarity of the correction is that the control station calculates the corrections in the pseudo-range between the control station and all satellites located above the radio horizon. To do this, measure the pseudo-ranges to all satellites and find the calculated pseudo-ranges from the reference coordinates of the control station and the coordinates of the satellites. These pseudo-ranges are taken as true. Differences between measured and calculated pseudo-ranges are transmitted to consumers in the form of corrections. Each user chooses the optimal constellation and the measured pseudo-ranges are corrected by the corrections received from the control station.

\section{Phase differential method}

With relative definitions, navigation measurements are performed using the same constellations of satellites at coinciding or close moments from two points. Based on the results of these measurements, the geocentric coordinates of these points are calculated. Subtraction of the same location components allows you to determine the projection of the baseline connecting the points.

Distance between points is calculated as:

$$
D=\sqrt{\Delta x^{2}+\Delta y^{2}+\Delta z^{2}} .
$$

To ensure the processing of measurements, it is necessary to have a permanent connection between the points in order to transfer the received data from one point to another or from both points to the object of joint processing in real time.

To improve the accuracy, measurements are accumulated in the form of arrays and further processed together. The advantage of relative definitions is that a number of systematic errors are compensated for when calculating the baseline, which makes it possible to more accurately determine the relative location of points.

Realization of the mode of relative determinations requires precise frequency and time synchronization of the receivers at points 1 and 2. Practically, at the moment of measurements, there is an initial displacement of the time scales, as well as their drift, leading to errors. Compensation for these errors is carried out by using differential measurements of the recorded signal parameters, especially in phase measurements.

When calculating the distances between points, mainly double phase differences of carrier waves are included in the measurement processing.

The measurement of the phases of carrier waves is accompanied by ambiguity in the definitions of an integer number of phase cycles.

Unambiguous registration of the phase is carried out within the phase cycle.

When measuring pseudo-ranges of the order of $20200 \mathrm{~km}$, reliable determination of the number of $20 \mathrm{~cm}$ wavelengths is a problematic task.

To find an integer number of wavelengths (phase cycles), there are several ways to resolve ambiguity, many of which are quite complex and time consuming [9-15].

In particular, the method based on the redundancy of phase measurements uses phase measurements on one or two frequency channels with simultaneous observations of five or more satellites. The unknown are the coordinates of the point being determined and the values of whole phase cycles. The joint solution of the constructed equations by the iteration method leads to finding the unknowns. 
The most practical is a method based on a combination of phase and code measurements, which is essentially step-combinational. As noted earlier, pseudo-range measurement is performed when the $\mathrm{C} / \mathrm{A}$ and $\mathrm{P}$ codes are turned on, which provides a preliminary determination of the distance with an error of 30 to $60 \mathrm{sm}$.

\section{CONCLUSION}

To achieve the goal of the article, the following was done:

the principles of GPS operation are considered;

analyzed the main operational characteristics of the satellite navigation system;

investigated alternative ways of organizing a satellite radio navigation system in order to improve operational characteristics;

the calculated operating costs of a GPS satellite.

It can be concluded that the main operational characteristics are related to the accuracy of determining the coordinates of the receiver. With the current state of the art technology, improved performance is expected in the areas of satellite life, data processing techniques and information transfer methods.

In connection with the plans for the modernization of the GPS system, we will mention what the main opportunities it will open to increase accuracy. Two new additional coded civil signals ( $\mathrm{C}$ / A code on L2 and a new signal on L5) will improve the user experience for high-precision measurement applications on long and short baselines, such as aircraft precision approach and automatic landing, mapping, geodetic and geophysical measurements, since the time of ambiguity resolution will decrease and the length of baselines will increase, which will allow using the best ionospheric correction at large distances. These actions will improve the accuracy of autonomous positioning. For scientific and geodetic measurements that do not use real-time, the centimeter level of accuracy will be achieved faster and at lower cost than now. This will be possible by using three frequencies to facilitate the disambiguation process when performing highly accurate phase measurements. It also reduces the chances of any accidental interference with the GPS system.

Scientists and engineers are already working to create a third generation GPS system. Due to the use of satellite communications and the installation of more powerful computing systems on them, the capabilities of the system will significantly expand.

\section{REFERENCES}

1 K. Oduan, B. Guinot. Time measurement of GPS base. Moscow: Technosphere, 2002.

2 Lipkin I.A. Satellite navigation systems. Moscow: 2006.
3 E.L. Afraimovich. GPS-monitoring of the Earth's upper atmosphere. Irkutsk: GU NTs RBKh VSNTs SO RAMS, 2006, $479 \mathrm{p}$.

4 A. V. Koshelev, A. K. Sinyakin. Physical principles of GPS / GLONASS operation. Monograph, Novosibirsk: $S G G A, 2009$, pp.110 .

5 A.A. Gennicke, G.G. Pobedinsky. Global satellite positioning systems and their application in geodesy. M., 2004.

6 A.V. Koshelev. To the determination of the refractive index of the atmosphere for high-precision geodetic measurements. Novosibirsk: SGGA, 2010, pp. 23.

7 Yu. A. Golikov. Calculation of the main technical and economic indicators of the work of a small enterprise. Novosibirsk, SSGA, 2004, pp. 17.

8 M. Iasechko, M. Kolmykov, V. Larin, S.Bazilo, H. Lyashenko, P. Kravchenko, N. Polianova and I. Sharapa. Criteria for performing breakthroughs in the holes of radio electronic means under the influence of electromagnetic radiation, ARPN Journal of Engineering and Applied Sciences, 15(12), 2020, pp. 1380 - 1384.

9 O. Sotnikov, M. Iasechko, V. Larin, O. Ochkurenko, and D.Maksiuta. The model of a medium for creation of electric hermetic screens of the radio electronic means, IJATCSE. $\quad 8(2), \quad 2019, \quad$ pp. 300-304. doi:10.30534/IJATCSE/2019/32822019.

10 M. Iasechko, O. Tymochko, Y. Shapran, I. Trofymenko, D. Maksiuta, and Y. Sytnyk. Loss definition of charged particles in the discharge gap of the opening of the box-screens during the formation of a highly conductive channel, IJATCSE. 8(1.3), 2019, pp. 1-9. doi: 10.30534/ijatcse/2019/0181.32019.

11 M. Iasechko, V. Larin, O. Ochkurenko, S. Salkutsan, L. Mikhailova, and O. Kozak. Formalized Model Descriptions Of Modified Solid-State Plasma-Like Materials To Protect Radio-Electronic Means From The Effects Of Electromagnetic Radiation, IJATCSE. $8(3), \quad 2019, \quad$ pp. 393-398. doi: 10.30534/ijatcse/2019/09832019.

12 M. Iasechko, V. Larin, O. Ochkurenko, A. Trystan, T.Voichenko, A. Trofymenko, and O. Sharabaiko. Determining the function of splitting the charged particles of the strongly ionized air environment in the openings of the case-screens of radioelectronic means, IJATCSE. 8(1.3), 2019, pp. 19-23. doi: 10.30534/ijatcse/2019/0481.32019.

13 M.M. Iasechko, and O.M. Sotnikov. Protecting of radio electronic facilities is from influence of powerful electromagnetic radiation, Published by Izdevnieciba Baltija Publishing, Collective monograph, Riga, 2019, pp.283-299.

14 A. Syrotenko, O. Sotnikov M. Iasechko, V. Larin, S.Iasechko O. Ochkurenko, and A. Volkov. Model of Combined Solid Plasma Material for the Protection of Radio-Electronic Means of Optical and Radio Radiation, IJATCSE, 8(4), 2019, pp. 1241 - 1247. doi:10.30534/ijatcse/2019/33842019.

15 O. Turinskyi, M. Burdin, M. Iasechko, V. Larin, Y. Gnusov, D. Ikaev, V. Borysenko, and V. Manoylo. Protection of board radioelectronic equipment from the 
Valentin Chimshir et al., International Journal of Emerging Trends in Engineering Research, 8(9), September 2020, 5191 - 5196

destructive powerful electromagnetic radiation with the use of natural technologies, IJETER, 7(11), 2019, pp. 542 - 548. doi: 10.30534/ijeter/2019/2371120 19.

16 M. Iasechko, V. Larin, D. Maksiuta, O. Ochkurenko, I. Krasnoshapka, Y.Samsonov, H. Lyashenko, A.Zinchenko, and R.Vozniak. Model description of the modified solid state plasma material for electromagnetic radiation protection, IJETER, 7(10), 2019, pp. 376 - 382. doi: 10.30534/ijeter/2019/027102019.

17 O. Turinskyi, M. Iasechko, V. Larin, D. Dulenko, V. Kravchenko, O. Golubenko, D.Sorokin, and O. Zolotukhin. Model and development of plasma technology for the protection ofradio-electronic means of laser emission, IJATCSE. 8(5), 2019, pp. 2429-2433. doi:10.30534/IJATCSE/2019/85852019.

18 M.Iasechko, Y. Gnusov, I. Manzhai, O. Uhrovetskyi, V.Manoylo, A. Iesipov,O. Zaitsev, M. Volk, and O. Vovk. Determination of requirements for the protection of radio-electronic equipment from the terroristic influence by electromagnetic radiation, IJETER, 7(12), 2019, pp. 772 - 777. doi: 10.30534/ijeter/2019/077122019. 